山्यRANÇAISE

$>\mathrm{DE}$

$\stackrel{1=1}{\simeq}$ PÉDAGOGIE

\section{Revue française de pédagogie}

Recherches en éducation

$184 \mid 2013$

Varia

\title{
Une approche praxéologique de la connaissance dans le domaine de la formation. Éléments pour un cadre théorique
}
A pragmatic approach of knowledge acquisition in adult training. Elements for a conceptual framework

\section{Brigitte Albero et Christian Brassac}

\section{OpenEdition \\ Journals}

Édition électronique

URL : http://journals.openedition.org/rfp/4253

DOI : $10.4000 / \mathrm{rfp} .4253$

ISSN : 2105-2913

Éditeur

ENS Éditions

Édition imprimée

Date de publication : 15 décembre 2013

Pagination : 105-119

ISBN : 978-2-84788-521-7

ISSN : 0556-7807

\section{Référence électronique}

Brigitte Albero et Christian Brassac, « Une approche praxéologique de la connaissance dans le domaine de la formation. Éléments pour un cadre théorique ", Revue française de pédagogie [En ligne], 184 | 2013, mis en ligne le 15 décembre 2016, consulté le 10 décembre 2020. URL : http:// journals.openedition.org/rfp/4253; DOI : https://doi.org/10.4000/rfp.4253 


\section{Positions, débats et controverses}

\section{Une approche praxéologique de la connaissance dans le domaine de la formation. Éléments pour un cadre théorique}

Brigitte Albero et Christian Brassac

Depuis les années 1990, la formation est officiellement reconnue par les responsables politiques européens comme une clé essentielle d'accession à la "société du savoir ». Or, sous de multiples pressions et contraintes, ce domaine peine à sortir de modèles réducteurs de la connaissance, de l'apprentissage et du développement humain. Cette situation rend difficile l'articulation entre recherche théorique et pratiques sociales et limite la pertinence des réponses apportées aux demandes d'un secteur en constante évolution. Sur la base de théories existantes et de résultats de travaux empiriques antérieurs, le propos de cette contribution est de montrer que les sciences humaines et sociales (SHS) apportent des outils de compréhension de l'activité humaine susceptibles de soutenir un changement aussi bien des représentations que des pratiques. La première partie synthétise des apports qui montrent que le "savoir ", objet ou état cognitif, n'est séparable ni des processus individuels de " connaissance » des sujets ni des environnements sociaux qui le déterminent. En conséquence, dans le champ de la formation notamment, la recherche et l'action ne peuvent pas être détachées des projets socio-économiques, technologiques ou politiques qui les portent. La seconde partie explicite en quoi une approche non strictement cognitiviste des processus cognitifs de production et d'acquisition des connaissances permet de comprendre les difficultés rencontrées par la formation dans sa contribution pratique au projet d'une "société du savoir ». Constatant la persistance de modèles dominants mais dépassés et la pertinence de théories émergentes mais dispersées, la troisième partie passe en revue pour les rapprocher plusieurs théories et travaux issus de différents secteurs de recherche en SHS. Elle vise ainsi à montrer que les caractéristiques de l'activité humaine dégagées peuvent fournir au champ de la formation des bases conceptuelles, susceptibles d'améliorer la pertinence, la comparabilité et la cumulativité des résultats, tant théoriques que pratiques. Le terme "praxéologie de la connaissance " est finalement utilisé comme l'expression sémantique de ce construit à la fois épistémologique, théorique et politique.

Mots-clés (TESE) : société de la connaissance, travail intellectuel, éducation des adultes, théorie d'apprentissage, sciences sociales.

La production des connaissances ${ }^{1}$ et leur développement dans les activités de formation ${ }^{2}$ (initiale, continue, tout au long de la vie) sont devenus aujourd'hui des secteurs particulièrement sensibles pour les " sociétés du savoir " (Rodrigues, 2004 ; UNESCO,
2005). Ils suscitent à ce titre l'intérêt de multiples acteurs éducatifs et politiques, industriels et scientifiques, et, plus largement, citoyens. Le principe d'accès généralisé à l'information, aux savoirs et aux lieux de la formation constitue le présupposé principal d'un 
projet à visée démocratique. Pour se concrétiser, celuici implique la réalisation simultanée de deux types d'accès qui sont rarement différenciés : l'accès physique aux objets matériels et immatériels qui véhiculent et organisent le savoir par la mise à disposition des moyens techniques et humains nécessaires ; l'accès intellectuel en termes d'activité mentale efficace (dispositions cognitives et conduites, capacités et modes de connaissance), indispensables à l'assimilation et à l'exploitation des savoirs disponibles. Or, cette activité présuppose une mobilisation volontaire des intentions et un engagement dans des processus de connaissance bien plus difficiles à standardiser, à organiser et à développer que le seul accès physique aux environnements et aux objets. Cette activation dépend de multiples conditions socio-économiques et culturelles qui nécessitent des politiques cohérentes pour exister, s'actualiser dans des dispositifs et se maintenir dans la durée. Ces conditions constituent aujourd'hui l'enjeu d'un développement durable qui chercherait à se mettre davantage au service du développement, voire de la survie planétaire, qu'à celui de la seule concurrence économique. Dans cette perspective, un projet politique visant le développement d'une "société du savoir " adaptée et efficace devrait être capable de repérer, de comprendre et de réguler la nature et les conséquences des tensions et des impasses engendrées par la transformation systématique des patrimoines immatériels de la connaissance en savoirs marchands.

Depuis plus d'un siècle, les sciences humaines et sociales (SHS) produisent des concepts et des modèles permettant de décrire et de comprendre les dimensions multiples de l'intelligence ainsi que l'interdépendance des processus qui constituent la connaissance en tant qu'activité humaine globale. Depuis des décennies dans les neurosciences, les avancées de la recherche sur le fonctionnement cérébral ne cessent d'élargir et de complexifier la conception des interactions entre les composantes et les niveaux sensoriels, émotionnels et intellectuels, individuels et sociaux de l'activité mentale. Dans ces champs disciplinaires, les résultats démontrent de façon convergente et irréfutable qu'il est impossible de comprendre et d'améliorer les processus cognitifs individuels ni d'assurer leur partage et leur transmission sous forme de savoirs sociaux sans dépasser les conceptions réductrices - égocéphalocentrées ${ }^{3}$ ou strictement rationnelles - du fonctionnement humain. Ces travaux devraient avoir des incidences directes ou au moins servir de repères dans le domaine de la formation. Pourtant, on ne peut que constater la persistance des modèles fonctionnels centrés sur les objets, construits pour l'essentiel sur la définition rationnelle des objectifs, des produits et des procédures. Ces modèles réducteurs de la connaissance, qui traitent de manière implicite ou présupposent acquis les processus cognitifs des agents qui les mettent en œuvre, finissent par faire obstacle à la recherche aussi bien qu'à l'intervention sociale. Ils ont pourtant le grand avantage d'être relativement faciles à modéliser et à standardiser. Les décideurs économiques et politiques, pressés par l'urgence de l'action et les contraintes nationales et internationales, tendent donc à prendre plutôt appui sur les modèles qui offrent des solutions techniques et pratiques simples à évaluer, à dupliquer et à diffuser. Ils en arrivent ainsi à soutenir des modèles dépassés aux résultats limités, tout en reconnaissant par ailleurs que l'éducation et la formation sont devenues des enjeux décisifs dans l'avènement des "sociétés du savoir ". Une telle attitude est d'autant plus dommageable que les changements profonds entraînés en particulier par les technologies numériques nécessitent d'abandonner les modèles et représentations qui ne correspondent plus aux instruments, ni aux acteurs, ni aux enjeux des mutations planétaires contemporaines. Un bilan des acquis scientifiques s'impose, suivi d'un repositionnement épistémologique et théorique suffisamment partagé pour favoriser la production de travaux aux résultats non seulement additifs mais cumulatifs.

Dans cette perspective, cette contribution a pour objectif de rapprocher divers apports formulés par des disciplines concernées par la connaissance et la formation, en particulier dans les champs de recherche qui s'intéressent aux pratiques professionnelles (économie, éducation, information, communication, droit, gestion, management, santé, sport, travail social par exemple). Sans prétendre faire œuvre d'érudition et sans prétendre proposer une synthèse qui se voudrait exhaustive, l'objectif est, plus modestement dans une visée heuristique, de dégager des articulations possibles ayant pour but de mettre en valeur quelques fondamentaux épistémologiques et théoriques. Si cette première ébauche s'avérait pertinente pour le champ, une telle entreprise collectivement assumée permettrait de faire converger plus aisément les courants de recherche sur des problématiques communes et des résultats partageables. Les différentes orientations de recherche seraient ainsi amenées à contribuer dans une perspective transdisciplinaire à une anthropologie de la connaissance qui replace les savoirs produits dans la réalité multidimensionnelle de leurs conditions humaines de production et d'activité. Elles pourraient, par ce faire, dépasser la juxtaposition infinie de définitions réductrices des savoirs en termes d'objets mono-logiques mutuellement exclusifs ${ }^{4}$, se 
donnant ainsi les moyens de formaliser, de façon plus ouverte et plus explicite, les conduites et les phénomènes sociaux contemporains de connaissance. Sur des bases de référence commune, les divers courants de recherche pourraient enrichir des formations mieux appropriées à la réalité actuelle de la numérisation et de l'interconnexion planétaire, préparer les acteurs aux exigences des "sociétés du savoir » pour leur donner vie et efficacité, mais aussi fournir des repères pour orienter l'action dans la complexité et l'antagonisme des multiples dimensions individuelles et collectives, sociales et politiques qu'elles mettent en jeu.

La première partie du texte est motivée par cette conviction. Elle présente un ensemble d'analyses issues de la recherche qui explicitent les relations entre la notion de "sociétés du savoir » proposée par les instances politiques et la production scientifique du domaine.

Sur ces bases et en prenant appui sur des travaux empiriques et conceptuels antérieurs régulièrement cités non pas à titre exemplaire mais dans la tentative d'en expliciter les étapes et jalons, la deuxième partie vise à argumenter la pertinence d'une approche externaliste de la connaissance en tant qu'activité humaine. Sans se donner comme contradictoire, cette approche se situe cependant dans un continuum à l'opposé des conceptions strictement internalistes ou mentalistes de la cognition. Les travaux des pragmatistes anglosaxons (James, [1907] 2007 ; Mead, [1934] 2006 ; Dewey, [1938] 1993) et de la psychologie historicoculturelle russe (Vygotski, [1934] 1985) ont montré, tout au long du siècle dernier, que l'intelligence et la connaissance humaines sont des processus lents et progressifs d'élaboration, dépendants de l'action des individus en étroite interaction avec leur environnement physique, leur milieu social et les instruments matériels et symboliques propres à leur culture. En termes systémiques, cette dépendance réciproque peut s'interpréter comme un couplage dynamique d'interrelations à la fois sociales et techniques. Pour se transformer en heuristique utile, cette interprétation suppose à son tour une redéfinition profonde des théories et des modèles de la formation, qui tienne compte des acquis rappelés plus haut : à la fois lieu et instance sociale de construction cognitive fortement située et marquée par son ancrage temporel et spatial, impliquant et engageant la totalité de la personne humaine (biologique, psychologique, socio-économique, culturelle, éthique). C'est seulement à cette condition que la notion de couplage entre pragmatique individuelle de l'action, environnement socioculturel et instruments techniques peut se transformer en cadre d'analyse pertinent, à la mesure des bouleversements actuels.

La troisième partie présente de façon synthétique quatre théories dont l'articulation pourrait s'avérer féconde : les théories de l'activité, de l'action située, de la cognition distribuée, des acteurs-réseaux. En relevant de la même épistémologie, leur rapprochement permet d'étayer plus précisément une conception interactionniste de la connaissance et de la formation, en tant que construits distribués et situés et couplages finalisés d'entités hybrides, composées d'humains et d'artefacts matériels et symboliques.

Cette contribution conclut sur l'intérêt d'une approche praxéologique de la connaissance pour la formation. Cette approche se caractérise par une analyse de la connaissance en tant que pratique sociale (knowing as a practice). Cette pratique réunit des dimensions qui ont pu être considérées de manière séparée (intellect et corps, individu et société, cognition et émotion) et couple des entités (humaines et non humaines ${ }^{5}$ en tenant compte de la diversité des modalités de l'activité cognitive : naturelles (exploration, imitation, mémorisation, autorégulation) et instituées (instruction, entraînement, enseignement, conseil, accompagnement), replacées dans le temps et l'espace des sujets-acteurs et des institutions. À la fois compréhensive dans son épistémologie, spécifique à son champ d'application et adaptable à ses divers terrains, une telle approche devrait permettre d'appréhender sous toutes ses facettes la réalité des modèles, des pratiques et des enjeux portés par les projets politiques sous-jacents aux "sociétés du savoir ", à leurs promesses, leurs limites et leurs conséquences.

\section{RECHERCHE ET “ SOCIÉTÉS DU SAVOIR » : INTELLIGIBILITÉ ET RESPONSABILITÉ}

De nombreux enjeux sociétaux traversent ce qu'il est dorénavant convenu d'appeler l'économie de la connaissance (Foray, 2000 ; Rodrigues, 2004). II s'agit, en résumé, de décrire, comprendre, analyser et modéliser les pratiques qui se développent dans les organisations humaines. La valeur, la dynamique ou encore l'efficacité de ces organisations dépendent de plus en plus de la capacité de leurs acteurs à mobiliser, de façon raisonnée et réfléchie, des savoirs de toutes sortes dans des situations diversement prévisibles. L'étude de ces travailleurs du savoir et de leurs activités est donc au centre de travaux qui 
cherchent à conceptualiser ce que l'on appelle également l'économie de l'immatériel (Caspar \& Afriat, 1988 ; Lévy \& Jouyet, 2007) ou encore le capitalisme cognitif (Moulier Boutang, 2008). Par ailleurs, la mobilisation de savoirs génère des flux de connaissances dans les collectifs (Gaudin \& Hatchuel, 2002) qui englobent un ensemble de processus et de mécanismes organisationnels parmi lesquels : production, création, localisation, rétention, mémorisation, capitalisation, traduction, protection, circulation, transmission (Viginier, 2002).

La prise en compte de ces travaux sur le terrain des entreprises, des collectivités publiques et autres organisations est indispensable. Elle ne l'est pas moins dans la réflexion épistémologique, théorique et méthodologique car ces travaux soulèvent de nombreuses questions ${ }^{6}$. Ils conduisent notamment à observer les procédures d'apprentissage permanent dans lesquelles les travailleurs du savoir sont engagés, face à l'obsolescence toujours plus rapide de connaissances aux flux complexes, incertains et labiles qui exigent une veille, une mobilisation et une réactualisation sans relâche des compétences. Ils se trouvent donc mis en demeure de devenir des « apprenants permanents » (Linard, 1989 ; Carré, 2005), au point qu'ils finissent par devenir experts dans la capacité à développer les habiletés, stratégies, compétences et conduites leur permettant de se former à tout moment, au cours et en dehors de leurs activités professionnelles (Le Meur, 1998 ; Robin, 2003). Cette situation particulière produit un continuum historiquement inédit d'apprentissages dans et en dehors des institutions spécialisées (Albero, 2001), notamment sur les lieux mêmes de la pratique professionnelle, par exemple lors de situations de capitalisation de connaissances (Brassac, 2008a, 2008b, 2010). Cette construction sociale de ce qui s'apparente au projet de "sociétés du savoir " prend donc appui sur des pratiques intensives de production de biens immatériels à finalités d'abord industrielles et marchandes, pour lesquelles la formation des agents économiques constitue un enjeu majeur. Certains secteurs de recherche l'ont bien compris et investissent ce domaine des pratiques, par exemple : la recherche en intelligence artificielle qui travaille à la conception et à l'usage de dispositifs numérisés de partage et de diffusion de connaissances $^{7}$; le secteur des sciences économiques quand il rassemble les recherches relatives à la notion de connaissance ${ }^{8}$; le secteur éditorial de l'archivage numérique du patrimoine culturel et scientifique ainsi que la production plus récente des bases de données à des fins de commercialisation.
Dans les secteurs de l'éducation et de la formation en revanche, la recherche reste en deçà de ce que l'on pourrait attendre d'une discipline qui relève des SHS. Elle ne prend que peu en compte l'acquis des études sur les pratiques sociales qui appréhendent les projets politiques relatifs aux "sociétés du savoir " selon une logique moins technique, cognitive ou marchande que sociale. Portées par des acteurs essentiellement animés par des convictions de partage, de démocratisation et de développement humain par l'accès aux connaissances, ces pratiques ne cessent pourtant de susciter des travaux nombreux sur des sujets variés (apprentissage et formation continuée "tout au long de la vie ", processus d'auto-formation et capacité d'auto-direction des apprentissages, sentiment d'auto-efficacité en formation, apprentissages collectifs dans les réseaux collaboratifs et les communautés de pratiques, phénomènes d'inclusion ou d'exclusion liés aux modes d'organisation des environnements d'éducation, de formation et de travail, etc.). La diversité des résultats est stimulante mais elle reste dispersée ${ }^{9}$ entre autant d'orientations disciplinaires, théoriques ou méthodologiques, ne cumule pas et peine à rendre intelligible les caractéristiques de ce que l'on qualifie de "sociétés du savoir ».

La question qui se pose face à ce constat n'est pas nouvelle. C'est celle du " cadre organisateur » commun (Linard, 1989, 2001) sur lequel pourraient s'accorder les disciplines concernées par les implications de l'évolution technique et sociale des activités humaines. Ce cadre devrait permettre d'expliciter un positionnement de recherche alliant, de manière étayée et assumée, à la fois la distanciation scientifique et l'engagement en fonction de principes et de valeurs, selon la dialogie proposée en son temps par N. Elias ([1983] 1993). La nécessité de cette double exigence pour la recherche du domaine est travaillée de diverses manières selon les auteurs (Latour, 1984, 2001 ; Roqueplo, 1996 ; Stengers, 1997 ; Dalmedico \& Pestre, 1998 ; Pestre, 2002 ; Brassac, 2007a) mettant en évidence les liens - intentionnels ou non, explicites ou non - entre production de connaissance et projets sous-jacents de société. Qu'en est-il en effet de la responsabilité du chercheur lorsque son activité, qui vise d'abord l'intelligibilité des situations et des processus, est soutenue par des financements publics ou privés à des fins de transformation des dispositifs, des pratiques et des conduites humaines ? Comment peut-il conserver une distance critique dans le rapport direct qu'il entretient avec les secteurs politiques, économiques, industriels ? Dans la disjonction entre une recherche qui se met au 
service de projets marchands et une recherche qui les ignore, n'y a-t-il pas place pour une recherche capable d'expliciter et d'étayer un positionnement en accord avec un projet - lui-même explicite - de société ?

La complexification rapide et brutale des problèmes et des enjeux sociétaux liés à l'interconnexion numérique de la planète rend plus nécessaire que jamais de maintenir cette double exigence : la distanciation par le renouvellement des cadres épistémologiques et des modes d'objectivation, conceptualisation et instrumentation méthodologique, ainsi que de conduite des enquêtes sur le terrain ; l'engagement des chercheurs en tant que sujets et acteurs sociaux, selon une éthique de responsabilité (Jonas, [1979] 1990 ) en relation avec la place et la fonction qu'ils accordent à leur travail dans une société démocratique. Cependant, cette position ne peut tenir sans un renouvellement paradigmatique radical, fondé sur une autre conception de la connaissance : une conception étroitement couplée à l'action, individuelle et sociale, inscrite dans un espace et un temps précis, incorporée et finalisée dans le cours quotidien de l'activité des acteurs et médiatisée par les objets et les instruments contemporains (Linard, 1989 ; Brassac, 2008a, 2008b, 2010). Le paradigme pragmatiste apparaît cohérent avec une telle visée. Un tel positionnement qui reconnaît d'emblée l'interdépendance entre faits et valeurs, épistémologie et éthique (Putnam, [2002] 2004) est en mesure de redistribuer les rôles et les fonctions, en recréant des relations là où il y avait disjonction entre : théorie et pratique, connaissance et action, science et société. Si donc les travaux en ingénierie et en intelligence artificielle sont importants pour le développement de systèmes techniques performants, les travaux conduits en SHS dans des perspectives anthropologiques, sociales et historiques le sont tout autant pour comprendre les phénomènes humains de construction et de transmission des connaissances et prendre toute la mesure des conséquences des systèmes techniques sur les conduites et les cultures humaines.

\section{PERSPECTIVE PRAGMATISTE DU RAPPORT À LA CONNAISSANCE DANS UNE ÉPISTÉMOLOGIE DE LA PRATIQUE EN FORMATION ?}

Les relations qu'entretiennent connaissance et action sont un thème ancien de la philosophie, et chaque époque, comme chaque culture, les réexplore à sa manière, actualisant ainsi deux perspectives traditionnellement opposées dans le monde occidental. L'une, d'inspiration platonicienne, conçoit la connaissance (épistémè) comme découplée de l'action (praxis), du faire (techné et poiesis), mais aussi du jugement (phronesis) en tant que capacité délibérative dans l'action. L'autre, d'inspiration aristotélicienne, conçoit à l'inverse cet ensemble comme étant relié pour former une connaissance de l'action pratique (Frega, 2006a). Si la première perspective fonde le rapport traditionnel à la culture savante, la deuxième perspective a été régulièrement actualisée, enrichie et théorisée dans ses fondements (Kant, Hegel, Marx) puis reprise selon des orientations plus contemporaines (Gadamer, Arendt ou Habermas), mettant en valeur les relations que chaque période historique et que chaque culture établit entre ses manières et formes de penser et d'agir.

Le secteur de l'éducation et de la formation a beaucoup produit dans le sens d'une affirmation du lien entre développement des connaissances et capacité d'action du sujet, depuis les écrits et les créations de pédagogues célèbres (Makarenko, Montessori, Decroly, Freinet, parmi les plus connus), en passant par les écrits et les pratiques contemporaines de psychologues qui ont fondé une approche spécifiquement andragogique (Houle, Knowles, Rogers, par exemple), jusqu'aux productions plus récentes des sciences de l'éducation engagées concrètement dans l'action éducative (Meirieu, Pineau, par exemple) ou étudiant selon un grain très fin l'activité effective en situation d'apprentissage et de formation de sujets en interaction, à partir de diverses approches théoriques et méthodologiques (Barbier, Durand, Pastré, Sensevy par exemple). Les travaux plus expérimentaux de la psychologie ont régulièrement étayé nombre des intuitions formulées par les penseurs et les acteurs de l'éducation, en Russie (Vygotski, Léontiev), en Europe (Piaget, Wallon), aux États-Unis (Bruner, Bandura), confirmant la nature transformative de la connaissance lorsqu'elle est reliée à l'action et à l'interaction.

En sciences économiques et de gestion, A. Amin et P. Cohendet (2004) ont actualisé l'analyse de cette relation en proposant une cartographie des théorisations produites pour comprendre les dynamiques d'élaboration de connaissances et leurs architectures dans les organisations (notamment Argyris \& Schön, 1978 ; Nonaka \& Takeuchi, [1995] 1997 ; Cook \& Brown, 1999). La formulation de trois types de conceptions ("strategic-management ", " evolutionary-economics 》 et "social-anthropology-of-learning ") qui s'organisent 
autour de figures tutélaires (le « manager », la « routine » et la " community») est heuristique. Ils montrent comment les deux premières figures (manager et routine) s'appuient sur une représentation de la rationalité inspirée du modèle de A. Newell et H. Simon (1972 ; Simon, 1991). Dans cette perspective internaliste de la cognition, la connaissance considérée comme un état mental qui caractérise en propre le sujet (knowledge as a possession) est identifiée par quatre termes : stockage, codifiabilité, individualisme, possession. Le troisième type (community) envisage la connaissance comme produit d'une action et d'une pratique qui comporte sa part de socialisation, en prenant appui sur les corps et les émotions, ainsi que sur la médiatisation notamment par des objets, des artefacts, des outils ou des instruments. Cette différenciation s'appuie sur la polarité « possédé-agi » et prône l'abandon de la conception internaliste de la cognition appuyée sur une rationalité réduite aux seules capacités mentales des sujets. Elle plaide en faveur d'une conception externaliste de la cognition appuyée sur une rationalité socialisée et reliée à l'action, qui privilégie l'importance des pratiques dans l'élaboration des connaissances. Qualifiées de " mindbody-thing practices " (Amin \& Cohendet, 2004, p. 83), celles-ci sont considérées comme configurées à la fois par les intellects (mind) des sujets-acteurs mais aussi par leurs corps (body) et les artefacts présents dans leur environnement (thing). Pourtant, la remise en cause par les approches externalistes permet de concevoir la conduite humaine comme le produit-processus d'un système de relations réciproques entre le sujet et le monde (Mead, [1934] 2006) couplant encéphale, corps et environnement (Clark, 1997).

Ces analyses sont très proches de celles développées en sciences de l'éducation dès les années 1980 par un chercheur tel que M. Linard, à l'occasion de sa critique des modèles cognitivistes, informationnels puis logico-symboliques alors dominants ${ }^{10}$, appliqués à la production et à la transmission des connaissances. Ces analyses confirment que, loin de se réduire au traitement rationnel et à la communication d'informations, les connaissances se développent et se transmettent d'abord dans un contexte non seulement psycho-social, en fonction des relations que les sujets entretiennent avec autrui, mais également techno-sémiotique en fonction des relations que les sujets entretiennent avec les instruments techniques et symboliques disponibles dans leur environnement. $\mathrm{Ni}$ entités abstraites détenues à titre personnel, ni individuellement forgées de manière solipsiste, tous ces travaux depuis plus de cent ans convergent pour montrer que les connaissances sont d'abord le résultat des interactions entre humains : largement médiées par des non-humains, produits et supports d'interactions à la fois épistémiques et émotionnelles, elles constituent des réseaux denses qu'il n'est pas toujours aisé de catégoriser et de codifier.

Depuis quelques années, stimulées par les apports de l'anthropologie et de la sociologie des sciences et des techniques, les SHS connaissent un renouveau de l'intérêt pour les travaux des pragmatistes américains (Karsenti \& Quéré, 2004 ; Lavergne \& Mondémé, 2008), en particulier ceux de G. H. Mead (Brassac, 2005, 2007b) et de J. Dewey (Zask, 2012 ; Frega, 2006a, 2006b ; Sensevy, 2010, 2011), mais aussi ceux d'un philosophe tel que M. Polanyi (Frega, 2006a). Dans la même veine, en sciences de l'éducation, les travaux de M. Linard ont suivi sur une période de trente ans de recherche l'évolution épistémologique des technologies éducatives et ses incidences sur les modèles de connaissance et les usages en formation. L'ensemble de ces travaux convergent sur la proposition de développer ce qui relèverait d'une " épistémologie de la pratique " selon les termes mêmes de R. Frega (2006a). Potentiellement fédératrice, cette proposition concerne tous les chercheurs qui s'efforcent de concevoir des modèles et des analyses de type "non dualiste » dans le domaine.

Le développement d'une épistémologie de la pratique, transversale aux sciences qui s'intéressent à la connaissance et à son développement dans la formation, permettrait de partager et d'explorer de manière plus méthodique ce positionnement en tant que postulat n'ayant plus à être ni démontré, ni justifié. En conduisant à renoncer à la conception disjonctive entre théorie et pratique et entre pensée et action, l'épistémologie de la pratique permet de penser la connaissance non seulement dans sa dimension encyclopédique et érudite finalisée par sa validation ultime en savoirs, mais aussi dans sa dimension plus prosaïque de résolution de situations singulières (Frega, 2006a). Moment de conceptualisation et de formalisation provisoire dans le continuum pensée-action, la connaissance est conçue à la fois en tant que processus et produit dans son rapport à l'activité, que celle-ci soit en cours, en réflexion ou en suspension. Non seulement le produit intégré de tous les éléments matériels et humains qui la constituent (conceptuels, discursifs, instrumentaux, sociaux, etc.), elle est en même temps le processus même qui permet leur actualisation par transformation des énoncés, des objets et des actions de connaissance, mais aussi des acteurs et des actants ${ }^{11}$ de cette connaissance. Rompant avec les disjonctions théorie/pratique, pensée/action, un tel positionnement épistémologique trouve sa cohérence 
dans l'articulation de quatre théories socioconstructivistes interactionnistes (Linard, 1989 ; Brassac, 2007a) qui contribuent à l'étayer.

\section{DES THÉORIES DISPERSÉES INSCRITES DANS UN MÊME PARADIGME : AMORCE D'UN CADRE THÉORIQUE COMMUN ?}

Un certain nombre de théories en SHS présentent un intérêt pour la formation car elles ont l'avantage de prendre en compte trois dimensions fondamentales : le développement au sens de processus dynamique inscrit dans le temps et l'espace ; l'interaction humain-humain et humain-environnement ; la place des objets, artefacts, outils et instruments, qu'ils soient matériels, virtuels ou symboliques. Parmi ces théories, quatre d'entre elles présentent des points communs bien qu'issues de champs disciplinaires différents : la théorie de l'activité (Léontiev, [1975] 1984) ; la théorie de l'action située (Suchman, 1987) ; la théorie de la cognition distribuée (Hutchins, 1995 ; Lave, 1988) ; la théorie des acteurs-réseaux (Latour, 2006). Si leur articulation a déjà été montrée (Licoppe, 2008) et s'il a même été envisagé de les fédérer autour de la notion de "praxéologie de la conduite humaine " (Brassac, Fixmer, Mondada et al., 2008 ; Brassac, 2007b), il s'agit ici d'en rappeler succinctement l'intérêt afin de montrer, de manière synthétique, en quoi elles peuvent constituer l'amorce d'un cadre théorique commun aux orientations de recherche qui s'intéressent à la connaissance et à son développement dans et par la formation.

La théorie de l'activité, qu'en sciences de l'éducation M. Linard proposait en 1989 comme " cadre organisateur » de la recherche en éducation et formation, constitue l'une des suites des travaux de la psychologique historico-culturelle soviétique (Vygotski, [1934] 1985), tout en s'appuyant sur le pragmatisme américain et spécialement les travaux de J. Dewey et G. H. Mead (Engeström \& Miettinen, 1999). Selon cette théorie, l'activité humaine est un processus développemental dans lequel sujets humains et artefacts sont conformés par l'environnement physique et social, tout en le constituant simultanément. Sur la base de l'idée vygotskienne de médiation, A. Léontiev ([1975] 1984) puis Y. Engeström (2000) élargissent la réflexion à l'activité collective en proposant une approche ternaire de l'activité. En mettant en valeur les relations entre sujet, objet et artefact médiateur, ces approches intègrent le développement des individus et le changement organisationnel dans une vision constructiviste dynamique.

La théorie de l'action située est une des approches inspirées par la démarche ethnométhodologique (Garfinkel, 1967) et l'analyse conversationnelle (Sacks, Schegloff \& Jefferson, 1974). Elle est développée par L. Suchman (1987) qui s'intéresse, à l'instar de l'ensemble des chercheurs relevant des Workplace Studies (Luff, Hindmarsh \& Heath, 2000), à l'organisation des activités professionnelles dans les lieux de travail. C'est en étudiant la manipulation d'une photocopieuse qu'elle montre l'importance cruciale, à côté des productions discursives, des objets matériels et des éléments situés dans l'environnement de travail pour le déploiement des cours d'action tels que, par exemple, la résolution de problème. Elle est ainsi conduite à redéfinir radicalement le rôle des planifications, qui loin de déterminer l'action n'en constituent qu'une ressource, parmi d'autres.

La théorie de la cognition distribuée (Hutchins, 1995 ; Lave, 1988) développée par les anthropologues de la cognition conduit à envisager que les composants de l'activité cognitive ne se limitent pas aux représentations mentales mais incluent les structures sociales et culturelles, les corps et les objets techniques. Les processus cognitifs, non uniquement intracrâniens, sont distribués sur les artefacts de l'environnement et sur les autres membres du groupe social. Leur analyse relève d'une approche anthropologique qui place sur un même plan artefacts et sujets.

La théorie des acteurs-réseaux (Callon, 1986 ; Latour, 2006), issue de l'anthropologie et de la sociologie des sciences et des techniques, implique aussi de considérer de manière symétrique les entités engagées dans les processus collectifs, quelle que soit leur nature humaine ou non humaine. Dans le cours de la dynamique interactionnelle, les entités se configurent mutuellement. Ce faisant, émergent progressivement des réseaux hétérogènes, à la fois sociaux et techniques qui, lorsqu'ils sont suffisamment interconnectés, constituent une nouvelle entité, un nouvel acteur appelé acteur-réseau. Pour autant que l'on suive les acteurs et les artefacts d'une place ou d'un état à un autre et que l'on puisse les étudier de manière symétrique, cette approche met en lumière les processus de couplages humains/nonhumains, opératoires dans le cadre d'une analyse sociotechnique de l'activité collective, par exemple ceux qui organisent les dispositifs de formation (Albero, 2010a). 
L'intérêt de ces théories pour la production scientifique en éducation et formation a été depuis longtemps identifié même si elles n'ont pas toujours été rapprochées en ces termes (Linard, 1989, 1994a, 1994b, 2001 ; Brassac, 2007a, parmi d'autres chercheurs). Toutefois, la généralisation du numérique en rend la pertinence d'autant plus vive, et nombre de travaux dans le domaine font aujourd'hui référence à l'une ou l'autre de ces théories (Barbier \& Durand, 2006 ; Bronckart, 2006 ; Bronckart, Bulea, Fillietaz et al., 2004). Dans le paradigme qu'elles soustendent, les procédures de construction, production, acquisition, transmission et plus largement de circulation des savoirs sont conçues dans l'interrelation entre connaissant(s), apprenant(s) et non-humain(s) (Brassac, 2010). Rendues opératoires grâce à l'apport du concept d'énaction ${ }^{12}$ développé en biologie (Maturana \& Varela, 1994 ; Varela, Thompson \& Rosch, [1991] 1993), ces théorisations confirment le caractère anthropobiologique des rapports entre connaissance et expérience dans le couplage humain/environnement. Elles redonnent ainsi toute sa place à la dimension instrumentale dans une relation orientée par le développement du sujet et finalisée par son souci de contrôle de l'environnement. La prise en compte de cette double finalité (développementale et instrumentale) peut permettre de ré-ancrer le rapport entre connaissance et expérience dans chaque contexte historique et culturel.

Alors que le positionnement épistémologique développé ci-dessus permet de mettre en valeur le caractère dialogique des mécanismes interactionnels de construction conjointe de significations (Brassac, 2004 ; Sensevy, 2010, 2011), les quatre théories évoquées engagent à accorder, dans cette même voie, une attention particulière à la fonction configurante des artefacts dans l'espace de l'activité humaine (Brassac, 2001 ; Albero, 2010b).

Dans une perspective renouvelée en formation, les apprentissages ont été davantage analysés comme étant inscrits dans un dialogisme qui accorde un rôle important à l'apprenant ${ }^{13}$, en tant que coproducteur des situations d'interactions, mais aussi de la valeur sémiotique des contenus de ces échanges. Les courants de l'ergonomie cognitive et de l'analyse de l'activité ont largement contribué à ces avancées dans l'analyse des situations de formation (Durand, 2006 ; Barbier \& Durand, 2006 ; Zeitler, 2011 ; Guérin, 2012). Dans ces analyses pourtant, l'objet technique n'est pas particulièrement pris en compte. Plus largement, la place et la fonction des non-humains ${ }^{14}$ dans les processus de connaissance n'attirent pour l'instant pas une attention particulière de la part de la recherche dans le domaine. Réduits habituellement à une fonction ancillaire découplée de l'activité, ils sont pourtant amenés à jouer, à certaines conditions, un rôle important de médiation éducative et d'instrumentation des apprentissages, spécifiant, de ce fait, les rôles et fonctions de l'intervention humaine (Albero, 2003, 2010a, 2010b).

Dans cette perspective, les quatre théories évoquées ci-dessus étayent l'enquête empirique qui permet d'observer de tels phénomènes lorsque par exemple : l'activité est en partie configurée par l'environnement physique et social15 (théorie de l'activité) ; elle leur est fortement liée en étant localisée d'un point de vue temporel, spatial et interactionnel16 (théorie de l'action située) ; l'environnement prend en charge, de manière distribuée ${ }^{17}$, une partie de l'organisation de l'activité, jouant ainsi un rôle de médiation ${ }^{18}$ de sens et non seulement de médiatisation d'un message (théorie de la cognition distribuée) ; les configurations de dispositifs ${ }^{19}$, plus ou moins congruents ou conflictuels, mis en œuvre par les institutions d'éducation et de formation rendent opérantes des formes émergentes issues de jeux d'acteurs (humains, non-humains, hybrides) qui dépassent les intentionnalités, finalités, raisons d'agir des individus et des collectifs (théorie de l'acteur-réseau).

Des travaux récents en sociologie de l'action (Barbier \& Trépos, 2007) et en anthropologie des connaissances (Vinck, 2007 ; Poitou, 2007) fournissent des exemples d'intégration de ces avancées théoriques qui, en permettant de dépasser des positionnements épistémologiques tantôt naturalistes, tantôt constructivistes, renouvellent des concepts anciens (médiation, collectif, monde, format, etc.) et produisent des concepts plus adaptés (traduction, intéressement, attachement, prise, etc.). En argumentant en faveur de l'importance historique des évolutions techniques dans l'évolution des conceptions intellectuelles d'une période et d'une culture, de tels travaux montrent comment les avancées épistémiques contribuent en retour à modifier, dans un registre plus pragmatique, les représentations ordinaires de l'action.

En multipliant les artefacts cognitifs dans tous les domaines et de manière inédite dans l'histoire humaine, la période actuelle impose l'internationalisation des échanges et la vision planétaire des problèmes. Mais la complexification qui en résulte repose, de façon très différente par rapport aux périodes précédentes, la question de la dimension collective de l'activité, de son pilotage et donc, 
finalement, de son sens politique. En éducation et formation, comme dans les autres activités à finalité sociale, la prise en compte de la dimension politique des intentions et des incidences de l'action instrumentée dans des environnements donnés pour neutres ouvre des pistes d'investigation. Elle permet d'étudier les environnements numériques de l'activité en tant que dispositifs dans une perspective ternaire et trilogique (Albero, 2010c, 2012) : non seulement " fonctionnels » en tant qu'agencements stratégiques d'humains et de non-humains, mais aussi "idéels " car configurés par des idées, idéaux, normes et modèles épistémiques et axiologiques, mais aussi "vécus " par des acteurs incarnés, inscrits dans une histoire, des trajectoires et des intérêts individuels et sociaux (Albero, 2010c, 2012). Elle permet également d'étudier la manière dont le dispositif, compris dans cette tridimensionnalité, "instrumente »20, matériellement et symboliquement, les processus de formation et d'apprentissage individuels et collectifs d'un point de vue technique, informationnel, cognitif, métacognitif, social ou encore psycho-affectif (Albero, 2003 ; Albero \& Nagels, 2011). Les tendances du dispositif et ses modes d'instrumentation permettent ainsi de mettre en évidence des " configurations d'activité " qui ouvrent ou non des possibles, donnant des sens parfois opposés à des termes apparemment partagés qui relèvent en fait de pratiques très différentes ${ }^{21}$.

L'apport essentiel de ces différentes approches en éducation et formation permet d'élucider nombre des difficultés rencontrées et de mettre en évidence les différences importantes de pratiques et de résultats souvent masquées par la modernité des outils ou l'adhésion à des courants de pensée et d'action. Un autre apport important consiste à fournir aux décideurs des instruments d'analyse explicites, scientifiquement et techniquement fondés, permettant de discerner dans les activités de formation quels objectifs, moments et aspects sont standardisables, industrialisables, autrement dit " massifiables " et ceux qui ne peuvent pas l'être. Ces distinctions peuvent permettre de mettre au jour des projets très différents de société (Albero, 2003, 2010a, 2010b ; Linard, 2004).

\section{ÉLÉMENTS DE CONCLUSION : VERS UN CONSTRUIT TRANSDISCIPLINAIRE ADAPTÉ ?}

En articulant une diversité de travaux en SHS relevant de la même épistémologie, la rencontre de la conception "praxéologique " de la connaissance
(Brassac, 2007a) et de l'approche " sociotechnique » des environnements de formation (Albero, 2010b) propose un construit de nature transdisciplinaire. En constituant un sous-bassement épistémologique commun, ce construit permet d'offrir à l'enquête empirique une synthèse d'éléments théoriques articulés entre eux et qui ne sont plus à démontrer. Au plan méthodologique, ce construit est en cohérence avec les techniques qui se centrent sur l'analyse des pratiques effectives et du sens que les acteurs accordent à leur action, celles de la " théorie ancrée " (Glaser \& Strauss, [1967] 2010) par exemple ou de l'analyse de l'activité dans ses diverses orientations actuelles22 (Clot, 1999 ; Schwartz, 2000 ; Theureau, 2004 ; Pastré, 2006 ; Barbier \& Durand, 2006 ; Sensevy, 2011).

L'importance des transformations accélérées (scientifiques et technologiques, politiques et économiques, industrielles et professionnelles) au cours de ces deux dernières décennies, le basculement de civilisation lié à l'interdépendance généralisée et à la standardisation des modes d'échange et de communication qui lui est attaché, ainsi que les enjeux du projet lié au développement de "sociétés du savoir ", tous ces faits justifient une telle tentative épistémique dans le domaine de l'éducation et de la formation. Orientée par une centration anthropo-logique de l'activité, elle se justifie d'autant plus que la puissance de l'innovation technique contemporaine tend à effacer nombre de frontières (humain/non-humain, matériel/ immatériel, présence/distance, science/technique, etc.) et à accélérer l'obsolescence de l'information, remettant en question le statut relativement stable des savoirs dans leurs différences avec le processus de connaissance. Potentiellement fédérateur, cet ensemble épistémologique, théorique et méthodologique devrait permettre d'étudier des questions aussi cruciales que, par exemple, les caractéristiques : d'une formation initiale pour des générations qui vivent dans des environnements en constante mutation ; d'une formation professionnelle dans un monde très rapidement évolutif ; des processus de transmission intergénérationnelle de savoirs et savoir-faire mais aussi des manières d'être ensemble dans un monde cosmopolite confronté au changement permanent ; des modalités sociales d'actualisation individuelle et collective au cours de la vie ; du passage de l'information à la connaissance, de la connaissance au savoir, du savoir à l'intelligence de l'action ; de l'usage des produits et processus de l'industrialisation de biens immatériels; etc. Pour avancer sur de telles thématiques, il apparaît indispensable de dépasser le décalage croissant entre conceptions 
épistémiques de l'action fondées sur d'anciens modèles et environnements de l'activité effective dans leur réalité à la fois humaine et technique. L'étude des configurations effectives conduit à prendre acte des déplacements qui s'opèrent. Dans le domaine de l'éducation et de la formation, il s'agit de comprendre les processus contemporains de production et de circulation de la connaissance entre les générations, les groupes sociaux, les cultures et les époques, qui s'organisent et s'instituent selon des valeurs, des normes, des expériences.

Quatre recentrations épistémiques nécessitent alors d'être opérées, constituant un défi individuel pour le chercheur et collectif pour les réseaux scientifiques et professionnels.

II s'agit de passer de l'analyse des processus éducatifs et formatifs en eux-mêmes à l'analyse de la manière dont, dans les conditions effectives auxquelles sont confrontés les acteurs, des connaissances sont produites ou non, en explicitant la nature et le statut de ces connaissances, ce qui peut constituer une instrumentation facilitant leur développement (orientation de travail fondatrice des différents courants de recherche centrés sur l'analyse de l'activité).

II s'agit moins de comprendre comment s'opère la production de connaissance dans les lieux institués de l'éducation et de la formation que de développer des savoirs sur la manière dont l'ensemble d'une société permet aux individus qui la constituent de développer des connaissances dans l'ensemble de leurs activités ordinaires : le travail, l'engagement militant et/ou confessionnel, les loisirs, l'exploitation des médias, des jeux et des loisirs, etc. ${ }^{23}$

Il s'agit de s'intéresser moins à la connaissance comme objet de discours « à propos de " et qui, une fois instituée, se stabilise provisoirement en savoir qu'à la connaissance en tant que processus " en vue de ", c'est-à-dire celle qui - de manière en partie tacite, incorporée, partagée - permet d'agir de manière efficace, mais aussi éthique, par la prise de décision, la négociation du consensus, la transmission de valeurs 24 .

II s'agit enfin de comprendre comment ces processus sont également à l'œuvre dans les lieux mêmes de la production de savoirs institués 25 .

Les enjeux d'une telle recentration sont pluriels. Au plan de la société, le développement qualitatif et quantitatif de la connaissance constitue un enjeu stratégique dans la capacité de cohésion des organisations face à la complexification, l'incertitude, l'accélération, la gravité des obstacles à lever. Dans une pluridiscipline telle que les sciences de l'éducation par exemple, faire comprendre la nature non applicative de la production de connaissances à orientation scientifique vis-à-vis de l'activité d'expertise (conseil, formation de formateurs, diagnostic, évaluation) ou de l'activité éducative et formative proprement dite est particulièrement stratégique pour permettre aux institutions de jouer leur rôle.

Au plan des individus, l'outillage intellectuel et psychique relevant de cette compréhension peut permettre de développer une capacité d'actualisation permanente dans un environnement rapidement évolutif et, par conséquent, une capacité d'adaptation face à des réalités changeantes. Dans une pluridiscipline telle que les sciences de l'éducation par exemple, faire comprendre le passage discontinu et non applicatif entre les acquis de la formation initiale et les modes d'actualisation des connaissances et des compétences par la créativité de l'action en cours, dans la formation professionnelle continue et dans la formation personnelle tout au long de la vie, justifie l'intérêt de la généralisation dans les formations des acquisitions de conduites relevant de la dynamique d'enquête 26 , en termes de capacité à problématiser, à documenter et à instrumenter les observations, à les confronter à d'autres points de vue, à constituer du réseau, etc.

Au plan anthropologique, l'enjeu est double pour le moins : il consiste à maintenir l'inscription des individus et des sociétés dans le cours d'une histoire (humaine, culturelle et biographique) et d'une genèse (processus, situations, périodes, moments). II consiste également à conserver une orientation collective (projet politique et social) et individuelle (projet existentiel) réfléchie et débattue en fonction de critères de valeur, non seulement en relation à des référentiels de nature économique, cognitifs ou professionnel, mais aussi éthiques.

L'analyse des transformations des sociétés (culturelles, politiques, économiques, professionnelles, techniques) et des modalités qui produisent des connaissances (science, expertise, compétence) est régulièrement à l'étude depuis des décennies. En revanche, le statut et les formes que prennent ces connaissances lorsqu'elles sont instituées en savoirs sont moins étudiés, notamment dans la mise en valeur des transformations qu'ils subissent en fonction des environnements et des intentions pour lesquels ils sont mobilisés et, en conséquence, de leur éventuel pouvoir performatif. 
Or, la science ne se réduit pas à une dimension technique matérialisable en une technologie industrialisable, économiquement rentable. Elle est aussi une entreprise intellectuelle et culturelle, créative et créatrice de sens et de liens pour toute la société. Dans un domaine du social tel que l'éducation et la formation, l'objectivité, la vérité, la pertinence et la validité interne ne peuvent constituer les seuls critères de validation de l'intérêt d'une production de connaissance à orientation scientifique. Cette production est irrémédiablement liée à des enjeux de société, à un projet de nature politique et à des conduites de nature éthique. C'est bien l'articulation de ce double intérêt scientifique et axiologique qui fonde le renouvellement épistémique proposé dans cette contribution.
En réaffirmant un principe de continuité entre formes d'organisation politique et institutionnelle et conceptions épistémologiques et conceptuelles, un tel renouvellement des cadres de pensée ne peut être que réfléchi et travaillé en cohérence avec les perspectives émergentes d'une actualisation du concept de démocratie (Callon, Lascoumes \& Barthe, 2001) mais aussi d'une pratique scientifique plus civilisée (Stengers, 2003).

Brigitte Albero

brigitte.albero@univ-rennes2.fr Université européenne de Bretagne-Rennes 2, CREAD (EA 3875)

Christian Brassac christian.brassac@engees.unistra.fr Université Nancy 2, Codisant-Sitcom, laboratoire InterPsy

\section{NOTES}

1 Tout au long de ce texte, nous différencierons quatre termes qui renvoient à des processus reliés entre eux et inscrits chacun dans des temporalités différentes : 1) la connaissance, ensemble des processus cognitifs singuliers par lequel un individu développe sa capacité à comprendre des phénomènes ; 2) les connaissances, produits ou états cognitifs singuliers résultant des interactions de l'individu avec son environnement humain et non humain ; 3) le savoir, processus de normalisation des connaissances singulières par les usages, conventions et règles d'une société ; (4) les savoirs, produits, objets ou états collectifs résultant d'un processus, plus ou moins consensuel et institué, de validation des connaissances singulières, dans un domaine spécifique.

2 Nous entendons le terme formation dans son sens le plus large, à la fois action externe finalisée auprès d'un individu ou d'un collectif par une instance sociale (donner une forme) et processus interne d'apprentissage et d'actualisation de soi (se donner une forme). Ainsi comprise, la formation peut se définir comme la systématisation d'une modalité particulière de médiation sociocognitive à visée éducative, sous-tendue par des valeurs et des projets plus ou moins partagés (Albero, 2003, 2010a).

3 Nous empruntons ce terme à J.-C. Kaufmann (2001).

4 Par exemple : unités de traitement formel, processus élémentaires de cognition, circulation d'information, produits et services marchands, artefacts technologiques ou items fonctionnels de compétence.

5 Ce concept est emprunté aux travaux du courant de l'anthropologie des sciences et des techniques (Akrich, Callon \& Latour, 2006 ; Latour, 2006).

6 À titre d'exemple : les modes d'appropriation des technologies numériques par les organisations ; l'émergence d'une activité économique qui tend à se réduire aux métiers de service ; l'importance grandissante des pratiques d'innovation qui débordent le cadre des services de recherche et développement.

7 Par exemple, Interaction Design au sein duquel se placent le champ du Computer Supported Cooperative Work et plus précisément celui du Computer Supported Collaborative Learning (Kaptelinin \& Nardi, 2006).

8 Par exemple, les travaux de A. Amin et P. Cohendet (2004); pour un compte rendu étendu, Brassac (2007a).
9 Cette limite du champ de recherche portant sur la formation a été étudiée plus particulièrement dans son rapport aux technologies : Albero, 2004 ; Albero \& Thibault, 2009.

10 La mise en cause du paradigme de la connaissance en tant que système rationnel de traitement de l'information a suscité pendant plus de trois décennies des débats de fond très vifs, notamment en intelligence artificielle. Ces débats ne sont pas encore totalement clos (Cf. Linard, 1989 ; Brassac, 2006b).

11 Conceptualisé en linguistique par A. J. Greimas (1966) pour définir un élément abstrait (être ou chose) défini par une structure de relations participant d'une manière ou d'une autre (active ou passive) à un procès, là où l'acteur est plutôt une entité concrète. Ce terme a été repris par les tenants de la théorie des acteurs-réseaux (voir plus bas) pour désigner " toute entité dotée de la capacité d'agir, c'est-à-dire de produire des différences au sein d'une situation donnée, et qui exerce cette capacité " (Akrich, Callon \& Latour, 2006, p. 242). C'est en ce sens qu'il est employé dans le texte.

12 On peut consulter sur cette question le dossier proposé par la revue Intellectica (Brassac, 2006b) et l'ouvrage de Stewart, Gapenne et Di Paolo (2011).

13 Les sciences de l'éducation utilisent les termes de " centration sur l'apprenant » et de " renversement de perspective » (Carré, 2005) entre des échanges centrés sur le savoir de l'enseignant ou du formateur, ses objectifs, ses critères d'évaluation, ses modalités d'intervention et des échanges centrés sur les savoirs de l'apprenant, ses intentions, son projet de formation, ses critères d'acceptabilité de la performance, son profil d'apprenant. Dans ce cas, le syntagme " changement de paradigme " est utilisé pour mettre en valeur les modifications des représentations, conceptions, modèles, sémantiques, modalités d'action conséquentes (Albero, [1998] 2000).

14 Ce terme, emprunté aux travaux du courant de l'anthropologie des sciences et des techniques, se réfère, par exemple, à l'ensemble des objets techniques qui structurent l'activité de formation, qu'ils soient anciens (papier-crayon) ou modernes (espace numérique) ; industriels (manuels) ou artisanaux (photocopies) ; matériels (tableau mural), virtuels (tableau électronique) ou encore symboliques (catégories et concepts). 
15 L'exercice d'une pédagogie est lié à une organisation particulière de l'espace, du temps, des objets et autres artefacts, qui soustend un certain rapport à la connaissance et conditionne largement les modes d'interaction entre acteurs. Une pédagogie peut être centrée tantôt sur le savoir savant médiatisé par l'intervenant, tantôt sur les apprentissages cognitifs et métacognitifs des apprenants, tantôt sur la dimension formative d'acquisitions expérientielles. Le rapport à la connaissance de l'enseignant, même implicite, détermine directement ses choix concernant la transmission, l'acquisition et la validation des connaissancesobjets. Elles configurent ses actes en accordant une place et une fonction très différente aux dispositifs, aux méthodes et aux objets techniques (Albero, 2010a). Un autre rapport à la connaissance conditionne un autre mode d'interaction qui conduit à organiser autrement l'espace, le temps, les objets et autres artefacts et peut finalement produire une pédagogie opposée dans ses finalités et ses conséquences (Albero, 2010a).

Concevoir d'emblée l'acquisition de connaissance comme devant passer d'abord par une transmission de savoir savant puis par une application et ensuite par un entraînement individuel conduit à organiser des amphithéâtres selon le schéma cours magistraux/travaux dirigés ou mise en ligne d'information plus ou moins scénarisée/tutorat. Une conception qui privilégie l'expérience et la construction collective de connaissance par problématisation et confrontation de points de vue conduit à prévoir des situations d'interaction et de coformation dans des environnements ouverts et flexibles (médiathèque, centre de ressources, plateformes) en attribuant des places et fonctions complémentaires aux ressources humaines et matérielles en tant que référent, médiateur, conseil, entraîneur, etc.

16 Chaque acte de formation (instruction, transmission, enseignement, conseil, accompagnement, entraînement, etc.) est lié à un ensemble de circonstants particuliers et d'acteurs singuliers, c'est pourquoi des processus tels que la formation aux métiers de l'éducation et de la formation, la mutualisation des ressources et des compétences, la généralisation de l'innovation, voire l'industrialisation de supports et de procès, sont si complexes à mettre en œuvre. La part spécifique à chaque situation qui caractérise si fortement de telles activités de connaissance échappe régulièrement à toute tentative de modélisation.

17 Rôles redondants, complémentaires ou contraires, dans certains cas, des ressources humaines et matérielles.

18 L'organisation matérialisée d'un centre de ressources ou virtuelle d'une plateforme numérique fournit des catégorisations et oriente les activités, de telle manière qu'elle médie un certain rapport à la connaissance. Exemples contrastés sur les terrains de la formation : entre une structuration de l'environnement cloisonné de manière normative qui véhicule une seule conception de la connaissance ou une structuration ouverte qui prévoit une variété de possibles (salles ou centre de ressources, modules ou e-portfolio) ; entre une standardisation des modalités de travail qui normalise les manières de penser et de faire ou une flexibilité face à une diversité de possibles (accès à l'information, navigation, interactions, validation des acquis, etc.) ; entre une référence à une seule autorité qui véhicule un modèle de type sco- lastique ou une variété des références explicitant leur mode de validité ; entre des modalités uniformes d'interaction qui prédéterminent des rapports de domination ou une variété de modalités qui ouvrent la possibilité du dialogue et de l'autodétermination ; etc.

19 Les exemples sont multiples à tous les niveaux de la formation : formation des enseignants ; pédagogie de l'enseignement supérieur ; innovation pédagogique intégrant le numérique ; formation tout au long de la vie ; formation continue et permanente ; éducation des adultes ; e-learning ; formation ouverte et à distance ; etc. La diversité des dispositifs institutionnels dans ce domaine confronte des modèles parfois opposés entre héritages et actualisations, élitisme et massification, localisation et internationalisation, offres et demandes, public et privé, logiques industrielles et d'artisanat individuel ou groupé, etc. Les positions et jeux d'acteurs se trouvent débordés par des phénomènes émergents qui les dépassent en tant qu'individus, mais aussi en tant que collectifs (corporations disciplinaires ou statutaires, syndicats, associations professionnelles, etc.) expliquant des conduites supposées idéologiques (érudition positionnée dans les rangs conservateurs et pédagogie dans les rangs réformistes), apparemment contradictoires (résistance supposée des enseignants à la "nouveauté "), réitératives dans le temps (répétition des phases d'intégration des objets techniques les plus récents), faussement innovantes (intégration d'outils modernes au service de conceptions éducatives dépassées) ou empêchées (développement des pédagogies de l'autonomie et de l'émancipation)

20 Inspiré des travaux de P. Rabardel (1995), l'emprunt de ce concept est empiriquement et théoriquement étayé dans des travaux antérieurs, en particulier : Albero, [1998] 2000, 2003, 2010a, 2010b.

21 II a ainsi été montré (Albero, [1998] 2000) que le terme « autoformation " pouvait s'inscrire dans un continuum tendu entre deux pôles paradigmatiques entre "instruction » et " autonomie ». À l'intérieur de ce continuum, quatre types de tendances plus marquées ont été distingués (prescriptif, tutoral, coopératif, autonomisant), situés entre deux pratiques déjà repérées (enseignement et autodidaxie).

22 La note de synthèse de P. Champy-Remoussenard (2005) en fait une première étude.

23 Cette orientation de travail est privilégiée par les courants de recherche centrés sur les pratiques de formation dite " informelle » et « non formelle ».

24 Cette orientation de travail est commune aux courants de recherche en sciences de l'éducation qui s'intéressent à l'activité effective, au sens que les acteurs accordent à leur activité et à l'objectivation des pratiques pédagogiques.

25 Cette facette est moins explorée par les sciences de l'éducation et davantage par des champs de recherche tels que l'anthropologie et la sociologie des sciences et des techniques.

26 C'est la position qu'ont pu défendre le secteur de la formation des adultes ou celui de la formation des enseignants dans la mise en place de formations «par» la recherche. 


\section{BIBLIOGRAPHIE}

AKRICH M., CALLON M. \& LATOUR B. (2006). Sociologie de la traduction : textes fondateurs. Paris: Mines Paris; les Presses.

ALBERO B. ([1998] 2000). L'autoformation en contexte institutionnel : du paradigme de l'instruction au paradigme de l'autonomie. Paris : L'Harmattan.

ALBERO B. (2001). «Pratiques d'apprentissage dans et hors institution : une dialectique enfin possible dans les dispositifs émergents de formation ". Recherches en communication, no 15, p. 103-119.

ALBERO B. (2003). « L'autoformation dans les dispositifs de formation ouverte et à distance : instrumenter le développement de l'autonomie dans les apprentissages ». In I. Saleh, D. Lepage \& S. Bouyahi (coord.), Les TIC au cœur de l'enseignement à distance. Actes de la journée d'étude du 12 novembre 2002, organisée par le laboratoire Paragraphe, université Paris 8-Vincennes-SaintDenis, p. 139-159.

ALBERO B. (2004). "Travaux, interrogations et pistes de réflexion dans un champ de recherche éclaté ". Savoirs, no 5, p. 11-69.

ALBERO B. (2010a). «Une approche sociotechnique des environnements de formation. Rationalités, modèles et principes d'action ". Éducation et didactique, vol. 4, no 1, p. 7-24.

ALBERO B. (2010b). «Penser le rapport entre formation et objets techniques : repères conceptuels et épistémologiques ». In G. Leclercq \& R. Varga, Dispositifs de formation et environnements numériques : enjeux pédagogiques et contraintes informatiques. Paris : Hermès ; Lavoisier, p. 37-69.

ALBERO B. (2010c). « La formation en tant que dispositif : du terme au concept ». In B. Charlier \& F. Henri (dir.), La technologie de l'éducation : recherches, pratiques et perspectives. Paris : PUF, p. 47-59.

ALBERO B. (2012). «Approche trilogique des dispositifs en formation : pourquoi est-ce que les choses ne fonctionnent-elles jamais comme prévu ? ». In C. Gaux \& I. Vinatier (dir.), Outils pour la formation, l'éducation et la prévention: contributions de la psychologie et des sciences de l'éducation. Actes du colloque OUFOREP, organisé à Nantes les 6 et 7 juin 2011, p. 59-63.

ALBERO B. \& NAGELS M. (2011). « La compétence en formation. Entre instrumentalisation de la notion et instrumentation de l'activité ". Éducation-formation, no e-296, p. 13-29.

ALBERO B. \& THIBAULT F. (2009). " La recherche française en sciences humaines et sociales sur les technologies en éducation ". Revue française de pédagogie, no 169, p. 53-66.

AMIN A. \& COHENDET P. (2004). Architectures of knowledge. Firms, capabilities and communities. New York: Oxford University Press.

ARGYRIS C. \& SCHÖN D. A. (1978). Organizational learning: a theory of action perspective. Reading : Addison-Wesley.

BARBIER J.-M. \& DURAND M. (2006). Sujets, activités, environnements. Approches transverses. Paris : PUF.
BARBIER R. \& TRÉPOS J.-Y. (2007). « Humains et nonhumains : un bilan d'étape de la sociologie des collectifs ". Revue d'anthropologie des connaissances, vol. 1, no 1, p. 35-58.

BRASSAC C. (2001). «Formation et dialogisme : l'exemple d'un apprentissage situé et distribué ". L'Orientation scolaire et professionnelle, no 30/2, p. 243-270.

BRASSAC C. (2004). «Action située et distribuée et analyse du discours : quelques interrogations ». Cahiers de linguistique française, no 26, p. 251-268.

BRASSAC C. (2005). " La réception de George Herbert Mead en psychologie sociale francophone : réflexions sur un paradoxe ». Les Cahiers internationaux de psychologie sociale, no 66, p. 3-14.

BRASSAC C. (2006a). " Avant-propos : énaction, externalisme et internalisme, les modalités d'un débat ». Intellectica, no 43, p. 7-9.

BRASSAC C. (2006b). «Internalisme/externalisme ». Inte/lectica, no 43.

BRASSAC C. (2007a). "Une vision praxéologique des architectures de connaissances dans les organisations ". Revue d'anthropologie des connaissances, vol. 1, no 1, p. 121-135.

BRASSAC C. (2007b). "Qu'est ce qu'un acte ? La réponse de George Herbert Mead ». @ctivités, no 4 (2), p. 165177.

BRASSAC C. (2008a). " Apprendre pour (et à) capitaliser des connaissances : une étude de cas ». Travail et apprentissages, no 2, p. 9-24.

BRASSAC C. (2008b). "L'acquisition de savoirs comme activité située et distribuée ». Communication à la 1 re conférence francophone Gestion des Connaissances, Société et Organisations, organisée à Troyes les 13 et 14 mai 2008.

BRASSAC C. (2010). " Le triptyque "formant-objet-apprenant" ». In D. Adé \& I. Saint-Georges (dir.), Les objets dans la formation. Usage, rôles et significations. Toulouse : Octarès, p. 253-263.

BRASSAC C., FIXMER P., MONDADA L. \& VINCK D. (2008). "Interweaving objects, gestures, and talk in context ". Mind, Culture and Activity: An International Journal, no 15/3, p. 208-233.

BRONCKART J.-P. (2006). " Les conditions de construction des connaissances humaines ". In M. Carton \& J.-B. Meyer (dir.), La société des savoirs. Trompe-l'œil ou perspectives ? Paris: L'Harmattan, p. 27-47.

BRONCKART J.-P., BULEA E., FILLIETAZ I. et alii (2004). "Agir et discours en situation de travail ". Cahiers de la section des sciences de l'éducation, no 103.

CALLON M. (1986). "Éléments pour une sociologie de la traduction ». L'Année sociologique, no 36, p. 169-207.

CALLON M., LASCOUMES P. \& BARTHE Y. (2001). Agir dans un monde incertain. Essai sur la démocratie technique. Paris : Éd. du Seuil.

CARRÉ P. (2005). L'apprenance. Vers un nouveau rapport au savoir. Paris : Dunod.

CARRÉ P. \& CASPAR P. (2004). Traité des sciences et techniques de la formation. Paris : Dunod. 
CARRÉ P. \& MOISAN A. (2002). La formation autodirigée. Paris : L'Harmattan.

CASPAR P. \& AFRIAT C. (1988). L'investissement intellectuel : essai sur l'économie de l'immatériel. Paris : Economica.

CHAMPY-REMOUSSENARD P. (2005). " Les théories de l'activité entre travail et formation ". Savoirs, no 8, p. 42-50.

CLARK A. (1997). Being There: Putting Brain, Body, and World Together Again. Cambridge : MIT Press.

CLOT Y. (1999). La fonction psychologique du travail. Paris : PUF.

COLIN L. \& LEGRAND J.-L. (dir.) (2008). La formation tout au long de la vie. Paris : Economica.

COOK S. \& BROWN J. (1999). «Bridging Epistemologies: the Generative Dance between Organizational Knowledge and Organizational Knowing ». Organization Science, vol. 10, no 4.

DALMEDICO A. D. \& PESTRE D. (1998). « Comment parler des sciences aujourd'hui ? ". In B. Jurdant (dir.), Impostures scientifiques. Les malentendus de l'affaire Sokal. Paris : La Découverte ; Alliage, p. 77-105.

DEWEY J. ([1938] 1993). Logique : la théorie de l'enquête. Trad. par G. Deledalle (Logic: The Theory of Inquiry). Paris : PUF.

DURAND M. (2006). “Activité(s) et formation ». Université de Genève : Carnets des sciences de l'éducation.

DURAND M. \& BARBIER J.-M. (2003). « L'activité : un objet intégrateur pour les sciences sociales? ». Recherche et formation, no 42, p. 99-117.

ELIAS N. ([1983] 1993). Engagement et distanciation. Contributions à la sociologie de la connaissance. Trad. par M. Hulin (Engagement und Distanzierung). Paris : Fayard.

ENGESTRÖM Y. (2000). «Activity Theory as a framework for analysing and redesigning work ». Ergonomics, no 34/7, p. $960-974$

ENGESTRÖM Y. \& MIETTINEN R. (1999). « Introduction ». In Y. Engeström, R. Miettinen \& R.-L. Punamäki (dir.), Perspectives on activity theory. Cambridge : Cambridge University Press, p. 1-16.

ENGESTRÖM Y., MIETTINEN R. \& PUNAMÄKI R.-L. (1999). Perspectives on Activity Theory. Cambridge : Cambridge University Press.

FORAY D. (2000). L'économie de la connaissance. Paris : La Découverte.

FREGA R. (2006a). John Dewey et la philosophie comme épistémologie de la pratique. Paris : L'Harmattan.

FREGA R. (2006b). Pensée, expérience, pratique. Essai sur la théorie du jugement de John Dewey. Paris : L'Harmattan.

GARFINKEL H. (1967). Studies in Ethnomethodology. New York : Prentice-Hall.

GAUDIN T. \& HATCHUEL A. (2002). Les nouvelles raisons du savoir. La Tour-d'Aigues : Éd. de l'Aube.

GLASER B. G. \& STRAUSS A. A. ([1967] 2010). La découverte de la théorie ancrée. Stratégies pour la recherche qualitative. Trad. par M.-H. Soulet \& K. Oeuvray (The Discovery of Grounded Theory: Strategies for Qualitative Research). Paris : Armand Colin.
GREIMAS A. J. (1966). Sémantique structurale : recherche et méthode. Paris : Larousse.

GUÉRIN J. (2012). Activité collective, apprentissage et formation. Paris : L'Harmattan.

HUTCHINS E. (1995). Cognition in the wild. Cambridge : Massachusetts Institute of Technology Press.

JAMES W. ([1907] 2007). Le Pragmatisme. Trad. par N. Ferron (Pragmatism: A New Name for Some Old Ways of Thinking). Paris : Flammarion.

JONAS H. ([1979] 1990). Le principe de responsabilité : une éthique pour la civilisation technologique. Trad. par J. Greisch (Das Prinzip Verantwortung). Paris : Éd. du Cerf ; Flammarion.

KAPTELININ V. \& NARDI B. A. (2006). Acting with Technology. Activity Theory and Interaction Design. Cambridge : MIT Press.

KARSENTI B. \& QUÉRÉ L. (dir.) (2004). La croyance et l'enquête. Aux sources du pragmatisme. Paris : EHESS.

KAUFMANN J.-C. (2001). Ego. Pour une sociologie de l'individu. Paris : Nathan.

LATOUR B. (1984). Les Microbes. Guerre et paix. Paris : Métailié.

LATOUR B. (1991). Nous n'avons jamais été modernes. Essai d'anthropologie symétrique. Paris : La Découverte.

LATOUR B. (2001). Pasteur: guerre et paix des microbes. Paris : La Découverte.

LATOUR B. (2006). Changer la société. Refaire de la sociologie. Paris : La Découverte.

LAVE J. (1988). Cognition in practice. Cambridge : Cambridge University Press.

LAVERGNE C. \& MONDÉMÉ T. (dir.) (2008). «Pragmatismes ». Tracés, no 15.

LÉONTIEV A. ([1975] 1984). Activité, conscience, personnalité. Trad. par G. Dupond \& G. Molinier (Dejatelnost. Soznanie. Lichnost). Moscou : Éd. du progrès.

LE MEUR G. (1998). Les nouveaux autodidactes : néo-autodidaxie et formation. Lyon : Chronique sociale.

LÉVY M. \& JOUYET J.-P. (2007). L'économie de l'immatériel. La croissance de demain. Paris : La Documentation française.

LICOPPE C. (2008). «Dans le carré de l'activité : perspectives internationales sur le travail et l'activité ». Sociologie du travail, vol. 50, no 3, p. 287-302.

LINARD M. (1989). Des machines et des hommes. Apprendre avec les nouvelles technologies. Paris : L'Harmattan.

LINARD M. (1994a). «Vers un sujet narratif de la connaissance dans les modélisations de l'apprentissage 》. Intellectica, no 19, p. 117-165.

LINARD M. (1994b). « From learner's styles to learner's activity: lessons from various learner-centered research ". In R. Lewis \& P. Mendelshon (dir.), Proceedings of the IFIP TC3/WG3.3: Lessons from learning, p. 57-79

LINARD M. (2001). « Concevoir des environnements pour apprendre : l'activité humaine, cadre organisateur de l'interactivité technique ". Sciences et techniques éducatives, no 8/3-4, p. 211-238.

LINARD M. (2004). « Une technologie démocratique est-elle possible? ». Savoirs, no 5, p. 73-78. 
LUFF P., HINDMARSH J. \& HEATH C. (2000). Workplace studies. Recovering Work Practice and Informing System Design. Cambridge : Cambridge University Press.

MATURANA H. R. \& VARELA F. J. (1994). L'arbre de la connaissance. Paris : Addison-Wesley.

MEAD G. H. ([1934] 2006). L'esprit, le soi et la société.. Trad. par D. Cefaï \& L. Quéré (Mind, Self and Society from the standpoint of a social behaviorist). Paris : PUF.

MOULIER BOUTANG Y. (2008). Le capitalisme cognitif. La nouvelle grande transformation. Paris : Éd. Amsterdam.

NEWELL A. \& SIMON H. (1972). Human Problem Solving. Englewood Cliffs (États-Unis) : Prentice-Hall.

NONAKA I. \& TAKEUCHI H. ([1995] 1997). La connaissance créatrice. La dynamique de l'entreprise apprenante. Trad. de The Knowledge-Creating Company: How the Japanese Companies Create the Dynamic of Innovation. Bruxelles : De Boeck.

PASTRÉ P. (2006). “ Apprendre à faire ». In É. Bourgeois \& G. Chapelle (dir.), Apprendre et faire apprendre. Paris : PUF, p. 109-121.

PESTRE D. (2002). «Sciences, philosophie des sciences et politique : le constat d'une myopie ». Critique, no 661662 , p. 432-442.

POITOU J.-P. (2007). " Des techniques de gestion des connaissances à l'anthropologie des connaissances ". Revue d'anthropologie des connaissances, vol. 1, n० 1, p. 11-34.

PUTNAM H. ([2002] 2004). Fait/valeur : la fin d'un dogme et autres essais. Trad. par M. Caveribère \& J.-P. Cometti (The Collapse oh the Fact/Value Dichotomy, and other essays). Paris ; Tel-Aviv : Éd. de l'Éclat.

RABARDEL P. (1995). Les hommes et les technologies. Approche cognitive des instruments contemporains. Paris : Armand Colin.

ROBIN J.-Y. (2003). Biographie professionnelle et formation. Quand des responsables se racontent. Paris : L'Harmattan.

RODRIGUES M.-J. (2004). Vers une société européenne de la connaissance. La stratégie de Lisbonne (2000-2010). Bruxelles : Éd. de l'Université de Bruxelles.

ROQUEPLO P. (1996). Entre savoir et décision, l'expertise scientifique. Paris : INRA Éd.

SACKS H., SCHEGLOFF E. A. \& JEFFERSON G. (1974). «A simplest systematics for the organization of turn-taking in conversation ». Language, no 50/4, p. 696-735.

SCHWARTZ Y. (2000). Le paradigme ergologique ou un métier de philosophe. Toulouse : Octarès.
SENSEVY G. (2010). «Esquisse d'une pragmatique didactique ». Psychologie de l'interaction, no 27, p. 179-223.

SENSEVY G. (2011). Le sens du savoir. Éléments pour une théorie de l'action conjointe en didactique. Bruxelles: De Boeck.

SIMON H. (1991). « Bounded Rationality and Organizational Learning ». Organization Science, no 2/1, p. 125-134.

STENGERS I. (1997). Sciences et pouvoirs. La démocratie face à la technoscience. Paris : La Découverte.

STENGERS I. (2003). " Et si un jour les sciences devenaient civilisées... ». La Recherche, no 367, p. 67-70.

STEWART J., GAPENNE O. \& DI PAOLO E. (2011). «Enaction ». Toward a New Paradigm for Cognitive Science. Cambridge : Massachusetts Institute of Technology Press.

SUCHMAN L. (1987). Plans and situated actions. The problem of human/machine communication. Cambridge : Cambridge University Press.

THEUREAU J. (2004). « L'hypothèse de la cognition (ou action) située et la tradition d'analyse du travail de l'ergonomie de langue française ». @ctivités, vol. 1, no 2, p. 11-25.

UNESCO (2005). Rapport mondial de l'UNESCO : vers les sociétés du savoir. Paris : Éd. UNESCO.

VARELA F. J., THOMPSON E. \& ROSCH E. ([1991] 1993). L'inscription corporelle de l'esprit. Sciences cognitives et expérience humaine. Trad. par V. Havelange (The Embodied Mind: Cognitive Science and Human Experience). Paris : Éd. du Seuil.

VIGINIER P. (2002). La France dans l'économie du savoir : pour une dynamique collective. Rapport du Commissariat général du Plan. Paris : La Documentation française.

VINCK D. (2007). "Cet obscur objet de connaissances". Revue d'anthropologie des connaissances, vol. 1, no 1 . p. 5-10.

VYGOTSKI L. S. ([1934] 1985). Pensée et langage. Trad. par F. Sève (Myslenie i rec'). Paris : Messidor ; Ed. Sociales.

WENGER E. (1998). Communauties of practice. Learning, meaning, and identity. Cambridge : Cambridge University Press.

ZASK J. (2012). "Postface ». In J. Dewey, Expérience et Nature. Trad. par J. Zask (Experience and Nature, 1925). Paris : Gallimard.

ZEITLER A. (2011). Apprentissages interprétatifs. Interprétation en action et construction de l'expérience. Paris : L'Harmattan. 